\title{
Prévention du suicide: il faut améliorer les réseaux
}

Martin Eichhorn, secrétaire de l'Association suisse d'intervention de crise et de prévention du suicide (ASICS)

L'Association suisse d'intervention de crise et de prévention du suicide (ASICS) organise le 30 septembre 2004 à Bienne une session de formation continue qui proposera, face à la complexité du problème du suicide, une approche différenciée des différents moyens d'intervention et de leur mise en réseau.

\section{Deutsch erschienen} in Nr. 28/2004
Information et inscription: Barbara Weil

IPSILON

Direction

Elfenstrasse 18

CH-3000 Berne 16

Tél. 0313591108

Internet: www.ipsilon.ch
Le suicide et le comportement suicidaire sont choses fréquentes. En Suisse, une femme sur 100 et un homme sur 30 décède par suicide et un habitant sur 10 a été traité au moins une fois médicalement pour une tentative de suicide. Chez les jeunes où - selon des estimations officieuses - le taux des tentatives est particulièrement élevé, les idées suicidaires sont à considérer comme «normales» tant elles s'avèrent fréquentes.

Le suicide et le comportement suicidaire sont des phénomènes extrêmement complexes dont la genèse, résultant à la fois d'une prédisposition et de facteurs sociaux, requiert une analyse approfondie.

Seule une petite partie des interventions en cas de crise sont assumées par le système de santé.

Divers exposés feront mention des services d'aide disponibles sur l'internet ou par téléphone ou de ceux dispensés par les milieux scolaires, par la police et autres. Ces exposés seront complétés par une exposition d'affiches accompagnant les initiatives et projets de prévention du suicide actuellement en cours de réalisation.

Que pouvons-nous apprendre les uns des autres? Dans ce domaine où tant de mythes sont encore bien présents, il nous paraît très important de tirer parti du savoir disponible dans les disciplines les plus diverses. Car le diagnostic d'une dépression à lui seul n'est de loin pas suffisant pour saisir la complexité du problème. Il faut une connaissance approfondie des différents groupes de suicidants. En effet, le comportement suicidaire des hommes et des femmes, des jeunes et des personnes âgées, des migrants et des étrangers établis de longue date ont des causes différentes. Il importe de savoir non seulement comment intervenir en cas de crise, mais aussi comment entrer en contact avec ces personnes en détresse.
Il convient d'abord de se demander comment améliorer les réseaux existants ou même, tout simplement, comment en former de nouveaux. Dans cette optique, le premier pas consiste à apprendre à se connaître et à accepter qu'une approche différente n'est pas forcément fausse mais peut être complémentaire.

Le Prof. Jean-Louis Terra lancera la discussion parmi les participants à la session en évoquant l'expérience française de mise en réseau en matière de prévention du suicide. En Suisse, comment pouvons-nous concrétiser les projets de mise en réseau? Nous espérons beaucoup que les travaux de groupe de l'après-midi sauront nous donner une impulsion à cet égard. Il est même fort probable que ces contacts informels jetteront les bases de futurs réseaux, le but à long terme étant l'établissement d'une collaboration institutionnalisée. Dans cette perspective, une idée importante et réalisable serait par exemple de faire régulièrement appel à des experts d'autres disciplines pour des sessions de formation postgraduée et continue. S'il le fallait encore, l'Alliance contre la dépression de Nuremberg est bien la preuve que la mise en réseau peut être couronnée de succès. Nous sommes convaincus que celle-ci pourra également jouer un rôle primordial en Suisse dans la lutte contre les comportements suicidaires.

Dans cette optique, l'ASICS est heureuse d'organiser cette session de formation continue en collaboration avec IPSILON, la «Fondation pour la prévention du suicide en Suisse». IPSILON reprend l'idée de la mise en réseau à l'échelle des associations et des organisations, idée qui a conduit il y a plusieurs années au niveau des membres individuels à la fondation de l'ASICS.

Il vous arrive certainement au quotidien, dans votre activité de médecin ou même en privé, d'être confronté à des comportements suicidaires. Vos connaissances vous permettent de contribuer de manière importante à la prévention du suicide. Mais les limites sont vite atteintes malgré tout, et c'est dans le but de repousser quelque peu ces limites que nous vous invitons à participer à notre session de septembre prochain. 